\title{
Epitaxial Graphene: a new Material
}

\author{
Th. Seyller ${ }^{1,{ }^{*}}$, A. Bostwick ${ }^{2}$, K. V. Emtsev ${ }^{1}$, K. Horn ${ }^{3}$, L. Ley ${ }^{1}$, J. L. McChesney ${ }^{2}$, T. Ohta ${ }^{2,3,5}$, J. D. Riley ${ }^{4}$, \\ E. Rotenberg ${ }^{2}$, F. Speck ${ }^{1}$, \\ ${ }^{1}$ Lehrstuhl für Technische Physik, Universität Erlangen-Nürnberg, Erwin-Rommel-Str. 1, 91058 Erlangen, Germany \\ ${ }^{2}$ Advanced Light Source, E. O. Lawrence Berkeley National Laboratory, Berkeley, California 94720, USA \\ ${ }^{3}$ Department of Molecular Physics, Fritz-Haber-Institut der Max-Planck-Gesellschaft, Faradayweg 4-6, 14195 Berlin, Germany \\ ${ }^{4}$ Department of Physics, La Trobe University, Bundoora, Victoria 3083, Australia \\ ${ }^{5}$ Present address: Sandia National Laboratories, Albuquerque, NM 87185-1415, USA
}

Received XXXX, revised XXXX, accepted XXXX

Published online XXXX

PACS For example: 71.20.Ps, 74.25.Fy

*Standard is "Corresponding author" e-mail thomas.seyller@physik.uni-erlangen.de, Phone +49 $91318528335,+4991318528335$

Graphene, a two-dimensional sheet of $s p^{2}$-bonded carbon arranged in a honeycomb lattice, is not only the building block of fullerenes, carbon nano tubes (CNTs) and graphite, it also has interesting properties, which have caused a flood of activities in the past few years. The possibility to grow graphitic films with thicknesses down to a single graphene layer epitaxially on $\mathrm{SiC}\{0001\}$ surfaces is promising for future applications. The two-dimensional nature of epitaxial graphene films make them ideal objects for surface science techniques such as photoelectron spectroscopy, low-energy electron diffraction, and scanning probe microscopy. The present article summarizes results from recent photoemission studies covering a variety of aspects such as the growth of epitaxial graphene and few layer graphene, the electronic and structural properties of the interface to the $\mathrm{SiC}$ substrate, and the electronic structure of the epitaxial graphene stacks.

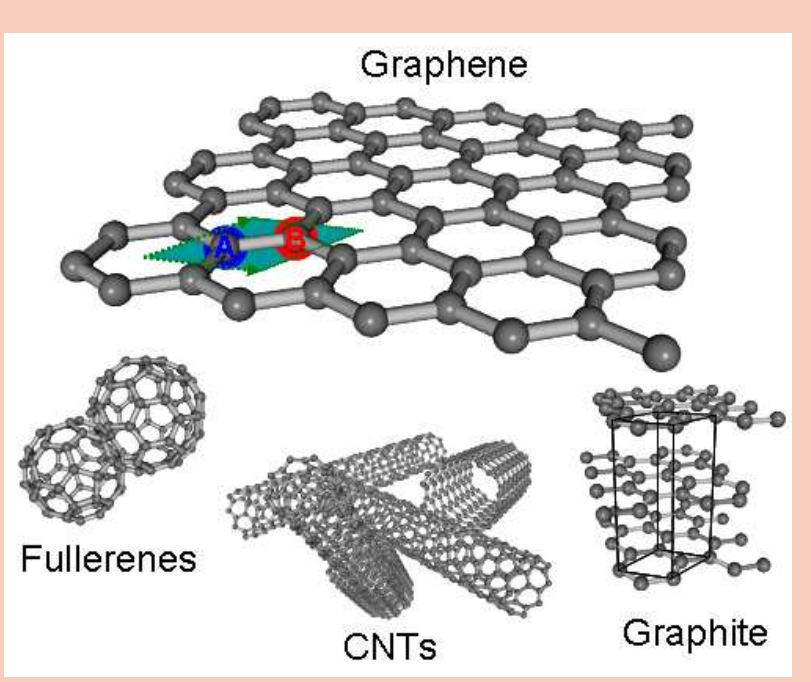

Copyright line will be provided by the publisher

1 Introduction The graphitization of $\mathrm{SiC}$ surfaces is a well known phenomenon: shortly after inventing a method (the Acheson process) to produce silicon carbide (then called carborundum) Edward G. Acheson [1] observed that heating of silicon carbide to very high temperatures leads to evaporation of $\mathrm{Si}$ and to the formation of graphite. This artificial graphite - frequently referred to as Acheson graphite - is extremely useful for lubrication purposes. It is clear, however, that for $\mathrm{SiC}$ as an electronic material graphitization of its surfaces which is observed to occur during processing steps such as post implantation annealing of post deposition annealing of contacts is most likely inconvenient. The role of graphite in contacts is discussed in a separate article in this volume [2]. On the other hand, it was shown in the pioneering work of Berger and coworkers [3-6] that ultra-thin layers of graphite grown carefully on $\mathrm{SiC}$ surface such that their thickness does not exceed a few monolayers has quite intriguing and potentially useful electronic properties.

A single monolayer of graphite is called graphene. It is a two-dimensional sheet of $s p^{2}$-bonded carbon atoms arranged in a honeycomb lattice and can be considered the building block of other carbon allotropes such as zero- 
(a) $\mathrm{SiC}(0001)$
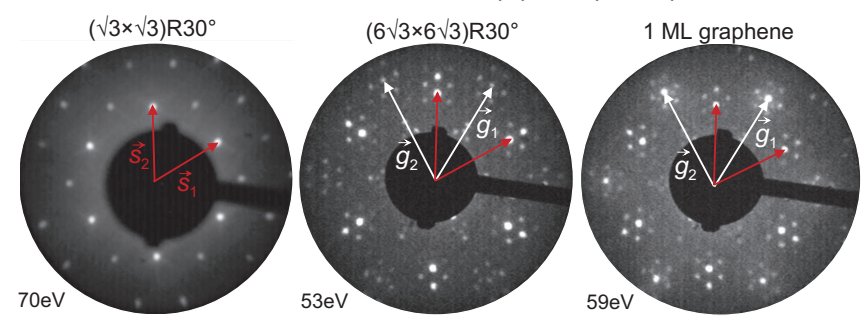

(b) $\operatorname{SiC}(000 \overline{1})$
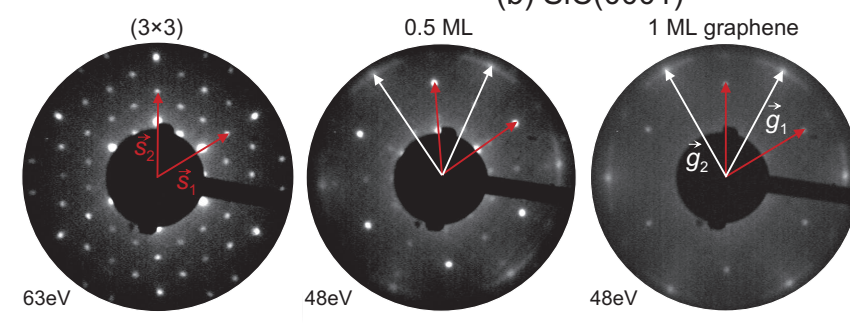

dimensional fullerenes ${ }^{1}$ [8], one-dimensional carbon nano tubes [9], and three-dimensional graphite. All these different modifications of carbon have been studied in detail both experimentally and theoretically [10-13]. Graphene, however, was primarily used as a theoretical model for studying the electronic structure of graphite [14-16] and carbon nano tubes $[12,13]$.

Recent progress in preparation techniques has enabled researchers to study the properties of graphene and of stacks of a few monolayers of graphene, so-called fewlayer graphene (FLG), experimentally. One method to fabricate FLG stacks is by graphitization of $\mathrm{SiC}$ surfaces as already mentioned above. Another method uses exfoliation of graphite to produce small flakes of graphene FLG $[17,18]$. Transport measurements on epitaxial graphene stacks on $\mathrm{SiC}$ [3-6] and of exfoliated graphene [18-22] have revealed their unusual transport properties. These arise (i) from the linear dispersion of the $\pi$ - and $\pi^{*}$-bands in the vicinity of the $K$-point of the hexagonal Brillouin zone and (ii) from the fact that the graphene unit cell contains two carbon atoms. As a consequence the electron wave function is a two component spinor wave function indication the probability to find the electron on either one of the two hexagonal sub-lattices. This can be seen as a pseudo-spin of the charge carriers [23]. As a consequence the behavior of the charge carriers is described by the Dirac equation [23] leading for example to the unusual quantum Hall effect [18-22]. Besides the interesting physics, graphene is also a promising material for high speed electronic devices $[3,4,24]$. Recently electron mobilities of up to $200.000 \mathrm{~cm}^{2} \mathrm{~V}^{-1} \mathrm{~s}^{-1}$ were demonstrated

\footnotetext{
${ }^{1}$ Note that in order to form graphene into fullerenes one has to build in five-membered rings, which in pure graphene must be considered as defects.
}

Figure 1 (Color online) Representative LEED patterns [7] of the polar SiC surfaces during preparation of graphene and FLG. The top row shows the $\mathrm{Si}(0001)$ surface. From left to right: $(\sqrt{3} \times \sqrt{3}) R 30^{\circ}$ reconstruction, $6 \sqrt{3}$ reconstruction, 1 monolayer (ML) graphene, few layer graphene with a thickness of ca. 5-6 monolayers. The bottom row corresponds to the $\mathrm{SiC}(000 \overline{1})$. From left to right: $(3 \times 3)$ reconstruction, $0.5 \mathrm{ML}$ graphene coexisting with the $(3 \times 3)$ and $(2 \times 2)_{\mathrm{C}}$ reconstructions, 1 ML graphene, FLG with a thickness of $4 \mathrm{ML}$. The reciprocal lattice vectors of the $\mathrm{SiC}\left(\mathbf{s}_{1}, \mathbf{s}_{2}\right)$ and graphene $\left(\mathbf{g}_{1}, \mathbf{g}_{2}\right)$ lattices are indicated. Note that the graphene diffraction spots on $\mathrm{SiC}(000 \overline{1})$ are smeared out azimuthally, indicating the presence of rotated domains. in free standing graphene [25-27]. Further applications are single molecule gas detectors [28,29], spintronics [30-32] or quantum computing [33]

Because epitaxial graphene is a purely twodimensional system located at the surface of its substrate $\mathrm{SiC}$ its properties are conveniently studied by surface science techniques such as low-energy electron diffraction (LEED), photoelectron spectroscopy in its different varieties, and scanning probe microscopy (SPM). Using these methods it is possible to study the growth of epitaxial graphene, its electronic and structural properties, as well as the interface to the substrate. Several such studies have been carried out by the present authors [7,34-43] and the main results will be summarized in this article.

2 Experimental aspects For the studies of epitaxial graphene on $\mathrm{SiC}$, on-axis oriented, nitrogen doped $6 \mathrm{H}-$ $\mathrm{SiC}(0001)$ and $(000 \overline{1})$ purchased from SiCrystal AG was used. The wafers had a doping concentration of $1.5 \pm$ $0.5 \times 10^{18} \mathrm{~cm}^{-2}$. Growth of graphene layers was achieved by solid state graphitization which requires heating the samples at temperatures above $1150^{\circ} \mathrm{C}$ until Si desorption commences and excess $\mathrm{C}$ is left behind on the surface. Except for the initial work [34] ex-situ hydrogen etching was used to obtain flat $\mathrm{SiC}$ surfaces. This procedure was carried out in a $\mathrm{SiC}$ chemical vapor deposition reactor at a hydrogen pressure of approximately $1 \mathrm{bar}$, a sample temperature of $1550^{\circ} \mathrm{C}$, and a flux of 3 standard liter per minute $(\mathrm{slm})$.

According to the phase diagram of polar $\operatorname{SiC}\{0001\}$ surfaces [44], there are two possible pathways for the growth of graphitic layers on $\mathrm{SiC}$. Both methods were carried out in our studies. One way is to start with an in situ cleaning step which removes oxygen and excess carbon from the surface by annealing it in a flux of $\mathrm{Si}$ as de- 
(a)

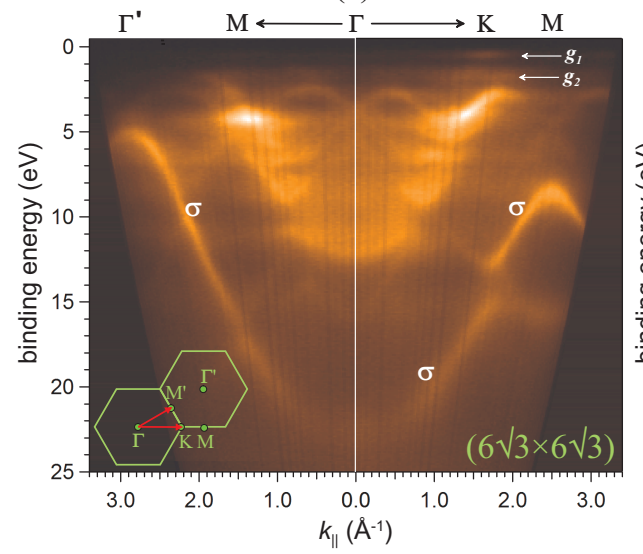

(b)

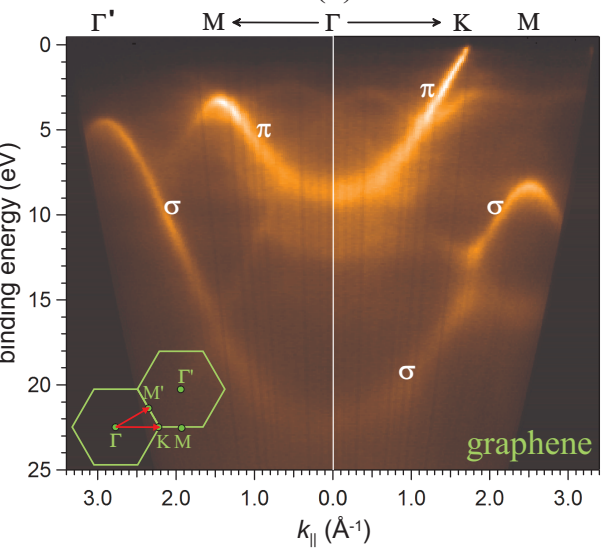

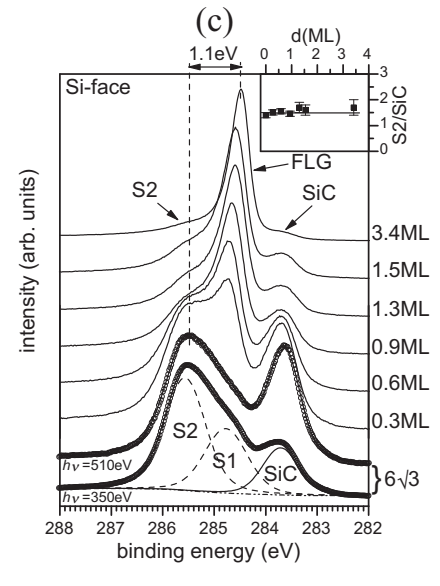

Figure 2 (a) and (b) Photoelectron intensity map vs. binding energy and parallel electron momentum of $\operatorname{SiC}(0001)-6 \sqrt{3}$ and one monolayer graphene on top of $\mathrm{SiC}(0001)-6 \sqrt{3}$, respectively [7]. Photon energy was $h \nu=50 \mathrm{eV}$. The insets shows the direction of $k_{\|}$within the hexagonal Brillouin zone of graphene.(c) C 1 s core level spectra of the $6 \sqrt{3}$ reconstruction and of FLG with increasing thickness ranging from 0.3 ML to 3.4 ML [7]. The inset shows the intensity ratio of component S2 and the bulk component SiC as a function of FLG thickness $d$. $d=0$ corresponds to the $6 \sqrt{3}$ reconstruction.

scribed elsewhere [34]. Then excess $\mathrm{Si}$ is gradually removed by subsequent annealing steps between at temperatures of up to $1400{ }^{\circ} \mathrm{C}$, until epitaxial graphene is formed. Depending on the surface polarity, a variety of reconstructions occurs during this treatment [44]. On $\mathrm{SiC}(0001)$ it is , $(\sqrt{3} \times \sqrt{3}) R 30^{\circ}, 6 \sqrt{3}$, and $(1 \times 1)_{\text {graph. }}$ On $\operatorname{SiC}(000 \overline{1})$ different reconstructions occur: $(2 \times 2)_{\mathrm{Si}},(3 \times 3),(2 \times 2)_{\mathrm{C}}$, and $(1 \times 1)_{\text {graph }}$, with the $(2 \times 2)_{\mathrm{C}}$ reconstruction appearing just in a small temperature region and in coexistence with the $(3 \times 3)$ and $(1 \times 1)_{\text {graph }}$ structures, respectively. Representative diffraction patterns are summarized in Fig. 1 [7].

The second pathway starts with the ex-situ H-etched surfaces which are covered with the silicate adlayer reconstruction [45]. Annealing of this reconstruction in vacuo induces desorption of oxygen and formation of the Si-rich $(\sqrt{3} \times \sqrt{3}) R 30^{\circ}$ structure and the $(3 \times 3)$ reconstruction on $\mathrm{SiC}(0001)$ and $(000 \overline{1})$, respectively. These are transformed into the carbon rich surface as described above [44].

Photoelectron spectroscopy measurements were carried out either at the storage ring BESSY II, using a toroidal electron analyzer for angle-resolved valence band studies or a hemispherical analyzer (Phoibos 150) for core level studies [7, 34, 35, 37, 38], or at the Electronic Structure Factory at beam line 7 of the Advanced Light Source $[36,39-43,46]$. For a detailed description the reader is referred to the original papers.

\section{Comparison of Graphene and FLG on $\operatorname{SiC}(0001)$ and $\operatorname{SiC}(000 \overline{1})$}

3.1 Film Structure determined by LEED Graphitization and thus growth of ultra-thin films of graphite is possible on both polar $\mathrm{SiC}\{0001\}$ surfaces. However, there are certain differences $[3,4,47-54]$. The most obvious one is the crystalline order which is evident from Fig. 1 which shows representative LEED patterns [7] of various stages of FLG growth on $\mathrm{SiC}(0001)$ and $(000 \overline{1})$. On $\mathrm{SiC}(0001)$ the FLG layers are aligned with respect to the substrate so that the primitive translation vectors of FLG and $\mathrm{SiC}$ enclose an angle of $30^{\circ}$.

The FLG layers on $\mathrm{SiC}(000 \overline{1})$ consist of rotationally disordered domains. This is witnessed by diffraction rings observed in low-energy electron diffraction (LEED). These rings, however, show a strong intensity modulation indicating a preference for certain rotational angles. There is also experimental evidence that the individual graphene layers in thicker FLG films on $\mathrm{SiC}(000 \overline{1})$ contain rotational stacking faults $[55,56]$. The misalignment on the substrate and the turbostratic structure of FLG films on $\mathrm{SiC}(000 \overline{1})$ was recently explained in terms of higher order commensurate structures of the growing graphene layers which leads to preferred but rotated orientations of the graphene layers [55].

Another important difference is the occurrence of a $(6 \sqrt{3} \times 6 \sqrt{3}) R 30^{\circ}$ reconstruction $(6 \sqrt{3}$ for short $)$ at the very beginning of growth on $\mathrm{SiC}(0001)$. This reconstruction remains at the interface between $\mathrm{SiC}(0001)$ and FLG $[7,38]$. The LEED pattern of monolayer graphene on $\mathrm{SiC}(000 \overline{1})$ shows no indication for a reconstruction of the substrate surface. This is a point of conjecture. Forbeaux et al. [51] concluded that growth of graphite on $\mathrm{SiC}(000 \overline{1})$ initially occurs on the $(2 \times 2)_{\mathrm{C}}$ reconstruction. However, LEED averages over a large area of the surface which could contain regions of different structure. Hass et al. [55] reported a long range order of the $(2 \times 2)$ reconstruction of $200 \AA$, which is at least ten times smaller than the coherence length of the graphene film. They suggested that different parts of their surface are in different stages of graphi- 
(a)

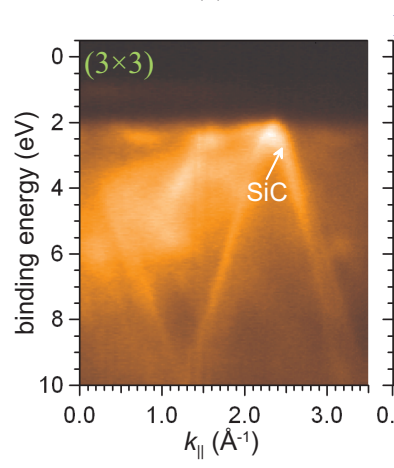

(b)

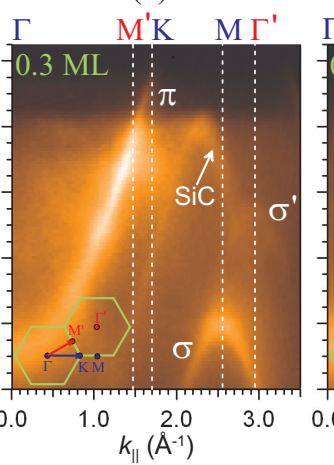

(c)

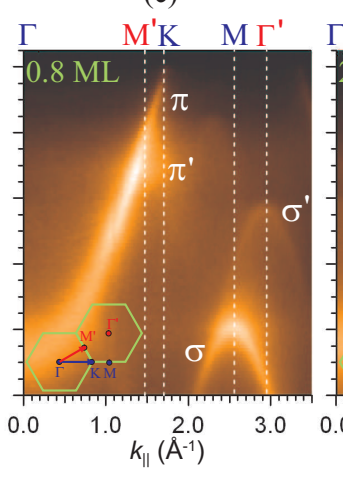

(d)

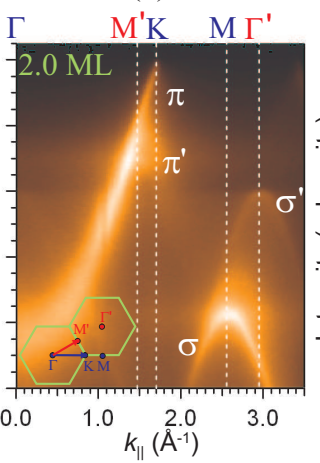

(e)

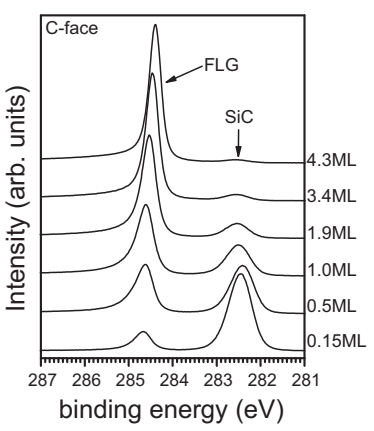

Figure 3 (a)-(d) Photoelectron intensity map vs. binding energy and parallel electron momentum acquired at different stages of FLG growth on $\mathrm{SiC}(000 \overline{1})$ starting from the clean $(3 \times 3)$ reconstruction [7]. The graphene overlayer thickness was determined from $\mathrm{C} 1 \mathrm{~s}$ core level intensities. (e) C 1s core level spectra taken at various stages of FLG growth on $\operatorname{SiC}(000 \overline{1})$.

tization. Also, the $(2 \times 2)_{\mathrm{C}}$ reconstruction is an adatom structure with Si adatoms $[57,58]$ in $H_{3}$ position. The presence of a $\mathrm{Si}$ adatom structure below the graphene layers, which form upon $\mathrm{Si}$ depletion of the surface, seems not very likely.

\subsection{Growth of graphene and FLG on SiC(0001)} studied by PES Fig. 2(a) and (b) show the valence band dispersion measured for the $6 \sqrt{3}$ reconstruction and $1 \mathrm{ML}$ graphene along the $\overline{\Gamma K}$ and $\overline{\Gamma M}$ azimuth of the graphene Brillouin zone, respectively. There are obvious similarities and differences to be noted. While the valence band structure of the $6 \sqrt{3}$ reconstruction exhibits clear graphenelike $\sigma$-bands which are well developed in band width and periodicity in momentum space it lacks the $\pi$-band of graphene. The well developed $\sigma$-bands indicate that the $6 \sqrt{3}$ reconstruction corresponds to an atomic arrangement of $\mathrm{C}$ atoms identical to graphene [7,38]. The $\sigma$-bands of the $6 \sqrt{3}$ reconstruction layer are shifted by $1.0 \pm 0.1 \mathrm{eV}$ towards higher binding energies with respect to those of neutral graphite. For monolayer graphene [Fig. 2(b)] this shift is reduced to $0.4 \mathrm{eV}$ with respect to graphite. This shift is caused by a partial filling of the $\pi^{*}$-bands due to charge transfer from the substrate, resulting in an n-type doping of the graphene layers such that the Dirac point $E_{D}$ shifts below the Fermi energy $E_{F}[36,39,39,43]$ as discussed below.

Instead of the $\pi$-band of graphene an accumulation of intensity is observed which is centered around $\Gamma$ and which has an envelope that strongly resembles the dispersion of the $\pi$-band. This was attributed to band folding caused by the potential of the large unit cell which affects the delocalized $\pi$-states more than the $\sigma$-states $[7,38]$. The bottom of this band at $\Gamma$ is located $3.2 \mathrm{eV}$ below the bottom of the $\pi$ band of graphene which points towards a covalent coupling of $p_{z}$-orbitals to the substrate [7]. A similar behavior was also observed for graphene on $\mathrm{Ni}(111)$ [59-62]. The $6 \sqrt{3}$ reconstruction is non-metallic, i.e. there are no states at the Fermi level. Two localized states $g_{1}$ and $g_{2}$ with binding energies of $0.5 \mathrm{eV}$ and $1.6 \mathrm{eV}$, respectively, are observed. The non-metallicity is of the $6 \sqrt{3}$ surface supports our conclusion that a strong interaction of at least part of the carbon $p_{z}$-orbitals with the substrate exists $[7,38]$.

Fig. 2(c) displays C 1 s core level spectra of the $6 \sqrt{3}$ reconstruction and of FLG films with increasing thickness. The spectrum of the $6 \sqrt{3}$ reconstruction contains a bulk component (SiC) and two surface components (S1/S2). Both surface components $\mathrm{S} 1$ and $\mathrm{S} 2$ stem from $\mathrm{C}$ atoms within a graphene-like layer [7], where one third of these atoms is bound covalently to the underlying $\mathrm{SiC}$ substrate leading to component S1. The other two thirds visible in the spectrum as component $\mathrm{S} 2$ are $s p^{2}$-hybridized and connected to C-atoms within the reconstruction layer only [7]. Interestingly, a shoulder due to the component $\mathrm{S} 2$ remains visible in the $\mathrm{C} 1 \mathrm{~s}$ spectra of FLG spectra of increasing thickness. It is attenuated in the same manner as the bulk component (see inset of fig. 2(c)) which is a clear indication that the $6 \sqrt{3}$ reconstruction remains at the interface between $\mathrm{SiC}(0001)$ and FLG [7,38].

3.3 Growth of graphene and FLG on SiC (0001) studied by PES Similar studies as described above were also carried out for $\mathrm{SiC}(000 \overline{1})$ [7]. Fig. 3(a)-(d) displays selected ARPES valence band spectra starting from the clean $\mathrm{SiC}(3 \times 3)$ reconstructed surface and ending with two graphene monolayers [7]. Already at a coverage of 0.3 ML the spectral signature of $\sigma$ - and $\pi$-bands of graphene is observed. Charge transfer leads to a rigid shift of all bands by approximately $0.2 \mathrm{eV}$ towards higher binding energy compared to graphite. The emission from $\mathrm{SiC}$ bulk bands is gradually attenuated and disappears almost completely for a coverage close to a monolayer (Fig. 3(c)). At that coverage, however, a fully developed band structure of graphene with undistorted $\sigma$-and $\pi$-bands is observed. At the same time C 1s core level spectra (see fig. 3(d)) taken at various stages of FLG growth on $\mathrm{SiC}(000 \overline{1})$ show no interface related component similar to the Si-face [7]. The spectra show only a bulk component and a component due to the 
(a)

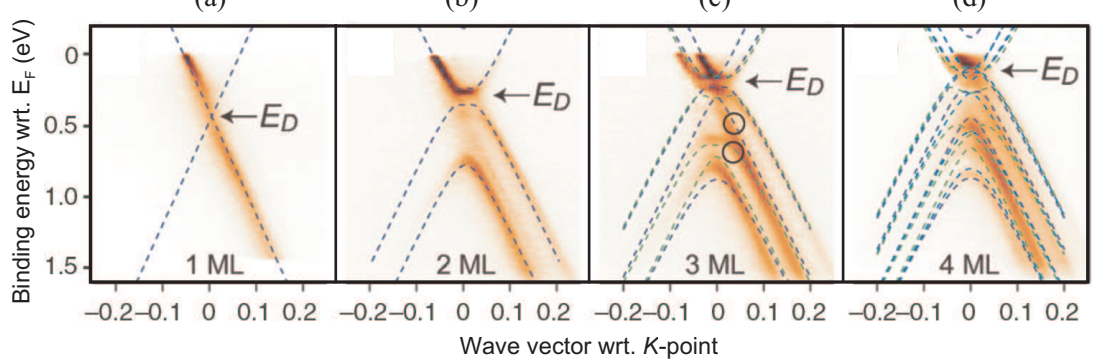

Figure 4 Evolution of the band structure close to the Dirac point while growing FLG with a thickness of up to 4 monolayers [43]. Note that the $\pi$-band of the monolayer is strongly renormalized due to many particle interactions [39]. Calculated bands for three configurations are shown: Bernal stackings $\mathrm{ABAB}$ andABAC (blue and light blue, respectively) and rhombohedral stackings (green). Adapted from [40]. growing FLG stack which - due to doping by the substrate - shifts from $284.65 \pm 0.05 \mathrm{eV}$ for the lowest coverage $(0.15$ ML) to $284.42 \pm 0.05 \mathrm{eV}$ for the highest coverage, which is characteristic for graphite. Note that the bulk component is found at a different binding energy $(282.5 \pm 0.05 \mathrm{eV}$ at a coverage of $4.3 \mathrm{ML}$ ) compared to the one observed on the Si face because of a different band bending [37]. Thus our photoemission data indicate a weak coupling of the FLG film to the $\mathrm{SiC}(000 \overline{1})$ surface. Note, however, that the signature of rotated domains, i.e., additional $\sigma$-band and $\pi$ band emissions labeled $\sigma^{\prime}$ and $\pi$ ', respectively, are visible at all coverages. This shows that graphene exists in rotated domains on the $\mathrm{SiC}(000 \overline{1})$ surface even at the very beginning of growth.

3.4 Discussion of FLG growth on Si-face and Cface From the data discussed above it is clear that the interface between epitaxial graphene and $\mathrm{SiC}$ depends on the surface orientation. For $\mathrm{SiC}(0001)$ pressing evidence is found for a graphene-like interface layer bound covalently to the substrate forming the $6 \sqrt{3}$ reconstruction. A similar model was proposed in theoretical studies [63-66]. This model structure consists of a covalently bound stretched graphene layer, where the $\mathrm{C}-\mathrm{C}$ distance had to be dilated by $8 \%$ in order to obtain a small enough unit cell that could be handled numerically. For both Si-face and Cface these studies find that the covalently bonded layer lacks graphene-like $\pi$-bands. The latter are obtained when a second graphene layer is placed on top of the covalently bound one, in agreement with the observations on the $\mathrm{Si}$ face [7]. What is different, however, is the presence of a dangling bond band in the calculations, which renders the surface metallic. As discussed elsewhere, this failure to describe the experimentally determined electronic structure correctly is most likely due to the wrong choice of surface unit mesh which leads to too many dangling bonds. On the C-face the model studied theoretically [63-66] does not apply at all: the experimental data do not give any indication for a covalently bound graphene layer. Instead it is found that graphene is bound by weak dispersion forces to the $\mathrm{SiC}(000 \overline{1})$ surface. As a possible explanation for the difference it was proposed [7] that the bonding of graphene to the $\mathrm{C}$-terminated surface in a fashion equivalent to the $6 \sqrt{3}$ interface structure is not possible because of the lesser flexibility of the $\mathrm{C}$ atoms in bond length and bond angle as opposed to the $\mathrm{Si}$ atoms on the $\mathrm{Si}$-face.
Another interesting aspect is the rotational order of FLG stacks on the two polar $\mathrm{SiC}\{0001\}$ surfaces. The rotational order observed on the Si-face is accompanied by strong covalent bonding of the bottom layer to the $\mathrm{SiC}$ substrate. On the C-face rotational disorder including rotational stacking faults $[55,56]$ is observed together with weak interaction between the substrate and the graphene layers. In order to account for that fact a growth model was proposed in which new graphene layers are formed at the bottom of the FLG stack [7]. On SiC(0001) this includes the $6 \sqrt{3}$ interface layer. On $\mathrm{SiC}(0001)$ each graphene layer starts out as the $6 \sqrt{3}$ interface layer which has an orientation imprinted by the covalent bonds to the substrate. Thus there is no freedom to form rotated domains and rotational stacking faults. Linear stacking faults, however, are possible and have been observed, indeed $[43,67]$. On the Cface, where the bottom graphene layer bonds only weakly by dispersion forces to the substrate, little constraints are acting on nucleating graphene layers, which allows for the observed $[55,56]$ rotational stacking faults.

3.5 Schottky barriers between SiC and FLG The barriers for charge carriers between $\mathrm{SiC}$ and FLG are of obvious importance if epitaxial graphene is to be used in electronic devices. The barriers were derived from core level photoelectron spectroscopy measurements on FLG films with thicknesses of 5-6 monolayers on $n$-type $6 H$ $\mathrm{SiC}(0001), p$-type $6 H$-SiC $(0001), n$-type $6 H$-SiC $(000 \overline{1})$, and $n$-type $4 H$-SiC $(0001)[2,35,37]$. The barriers observed by photoemission are in good agreement with those determined by electrical measurements [2].

The barrier for electrons on n-type $6 H-\mathrm{SiC}(0001)$ is rather small $(0.3 \pm 0.1 \mathrm{eV})$. For $n$-type $4 H$-SiC $(0001)$ the barriers is larger by $0.3 \mathrm{eV}$, which corresponds to the con-

Table 1 Schottky barriers $\Phi_{b}$ between FLG and SiC determined by photoelectron spectroscopy $[2,35,37]$. The error in $\Phi_{b}$ is $\pm 0.1 \mathrm{eV}$.

\begin{tabular}{ccccc} 
Polytype & $\begin{array}{c}\text { Surface } \\
\text { orientation }\end{array}$ & Doping & $\begin{array}{c}N_{D} \\
\left(\mathrm{~cm}^{-3}\right)\end{array}$ & $\begin{array}{c}\Phi_{b} \\
(\mathrm{eV})\end{array}$ \\
\hline $6 \mathrm{H}$ & $(0001)$, on-axis & $\mathrm{N}$ & $1 \times 10^{18}$ & 0.3 \\
$6 \mathrm{H}$ & $(0001), 3.5^{\circ}$ off-axis & $\mathrm{Al}$ & $1 \times 10^{16}$ & 2.7 \\
$6 \mathrm{H}$ & $(000 \overline{1})$, on axis & $\mathrm{N}$ & $1 \times 10^{18}$ & 1.4 \\
$4 \mathrm{H}$ & $(0001)$, on axis & $\mathrm{N}$ & $1 \times 10^{18}$ & 0.6 \\
\hline
\end{tabular}


duction band offset between the two polytypes. The barrier is also strongly face specific so that it is much larger on the $\mathrm{C}$-face of $n$-type $6 \mathrm{H}$-SiC. This could be an advantage of $\mathrm{C}$-face oriented $\mathrm{SiC}$ for building devices.

\section{Band structure of FLG near the K-point}

4.1 From graphene to graphite: evolution of the band structure The details of the electronic band structure in the vicinity of the $K$-point of the hexagonal Brillouin zone of graphene is of particular interest because it gives rise to the remarkable transport properties of graphene. Therefore a systematic study of the thickness dependent band structure of epitaxial graphene was carried out using ARPES [43]. Figure 4 demonstrates the evolution of the $\pi$-band in the vicinity of the Dirac point $E_{D}$ with increasing layer thickness. A complex band structure is observed. The position of the Dirac point $E_{D}$ below $E_{F}$ level is attributed to a charge transfer from the $\mathrm{SiC}$ substrate to the FLG layers. The position of $E_{D}$ with respect to $E_{F}$ can be varied systematically by adsorption of alkali atoms such as potassium, which result in a charge transfer to the graphene/FLG stack. The bands of the monolayer are strongly normalized, i.e. they deviate considerably from the expected linear behavior, due to many-body interactions as will be discussed below. The band structure of bilayer graphene on $\mathrm{SiC}(0001)$ shows a band gap of $0.15 \mathrm{eV}$, which is caused by a layer dependent onsite Coulomb potential and which can be controlled via doping of the top layer [36] as discussed further below.

In order to analyze the observed bands, they were modeled with a generalized tight binding Hamiltonian [43, 68-70].

$$
\begin{gathered}
\mathcal{H}=\left(\begin{array}{cccccc}
\alpha_{1} & \beta_{0} & & & \\
\beta_{0}^{\mathrm{T}} & \alpha_{2} & \beta_{s} & & \\
& \beta_{s}^{\mathrm{T}} & \alpha_{3} & \beta_{0} & & \\
& & \beta_{0}^{\mathrm{T}} & \alpha_{4} & \beta_{s} & \\
& & & \beta_{s}^{\mathrm{T}} & \ddots & \\
& & & & & \alpha_{\mathrm{N}}
\end{array}\right), \\
\alpha_{i}=\left(\begin{array}{cc}
E_{i} & v \pi^{\dagger} \\
v \pi & E_{i}
\end{array}\right), \quad \beta_{s}=\gamma_{1}\left(\begin{array}{rr}
0 & s \\
1-s & 0
\end{array}\right),
\end{gathered}
$$

where $E_{i}$ is the on-site Coulomb energy for layer $i$, $\pi=p_{x}+i p_{y}, \gamma_{1}$ is the interlayer hopping integral, and $v$ is the band velocity. The on-site Coulomb potential is caused by a layer dependent charge density [36, 43]. Different stacking orders are taken into account by the variable $s$ which is 0 for Bernal (ABA...) and 1 for rhombohedral (ABC...) stacking $[40,43]$. The reference energy is the Fermi level $E_{\mathrm{F}}$, and $E_{\mathrm{D}}=\operatorname{Tr}(\mathcal{H} / 2 N) . N$ is the number of layers. The total charge density $n$ in the FLG layer was determined by measuring the size of the Fermi surface. The best fit parameters are compiled in table 2 . The calculated

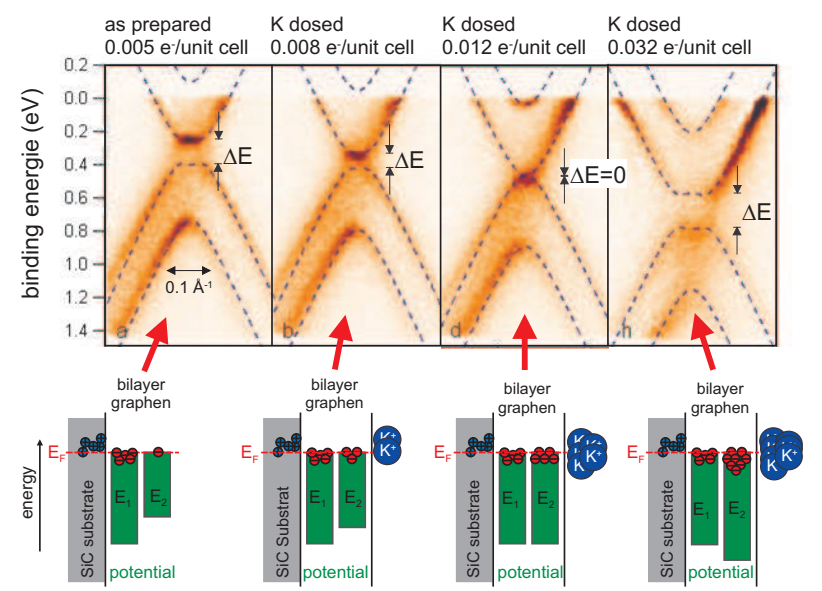

Figure 5 Closing and opening of the band gap in bilayer graphene induced by potassium adsorption [36] ontop of the bilayer stack. The left panel shows the as-prepared bilayer on $\mathrm{SiC}(0001)$. Charge transfer into the bottom layer gives rise to different on-site Coulomb potentials $E_{1}>E_{2}$ which results in a band gap. Doping of the top layer by potassium adsorption on the surface of the bilayer stack reduces the difference between the onsite potentials. If $E_{1}=E_{2}$ the gap is closed. Further $\mathrm{K}$ dosing increases the potential difference again $\left(E_{1}<E_{2}\right)$ which reopens the gap. The increasing shift of $E_{D}$ below $E_{F}$ is due to the increasing occupation of the $\pi^{*}$-bands.

band structures are in good agreement with the experimental data. In addition, parameters obtained in a recent STS study [67] are also listed in table 2 for comparison. A good agreement between the two studies is seen.

A comparison of the experimental band structure and calculated band structures for trilayer graphene on $\mathrm{SiC}(0001)$ led us to the conclusion that in this case domains with Bernal-type and rhombohedral-type stackings coexist [43] with equal weight. This is evident from the bands marked by the two circles in fig. 4(c). A recent STM and STS study of few-layer graphene on SiC(0001) [67] also found evidence for linear stacking faults, leading to rhombohedral $A B C$ stacking for the trilayer. The ARPES data also indicate that quadlayer is dominated by Bernaltype stacking, which may indicate that the second-nearest neighbor interaction is important for stabilizing the Bernal stacking in bulk graphite.

By analyzing the layer-dependent onsite Coulomb potentials $E_{i}$ it was possible to assign charge densities to the individual layers [43]. The total charge density amounts to about $1 \times 10^{1} 3$ electrons $/ \mathrm{cm}^{2}$. About $85 \%$ of this charge resides in the layer closest to the substrate, most of the rest in the second layer closest layer. From the charge distribution an in-plane screening length of $1.4 \AA$ and $1.9 \AA$ was determined for three and four monolayer thick epitaxial graphene films, respectively [43].

4.2 Switching the gap of bilayer graphene $\mathrm{Bi}$ layer graphene is a very special case of FLG. The presence 
Table 2 Best fit TB band parameters for $N=1-4$ layers graphene [43]: Fermi velocity $v$, is given in . The electron density $n$ is measured in $10^{-3}$ electrons per 2D unit cell. The Dirac point energy $E_{D}$, the onsite Coulomb potentials $E_{i}, i=1-4$, and the interlayer coupling constant $\gamma_{1}$ are given in eV. For comparison, the values obtained in a recent STS study [67] are shown as well.

\begin{tabular}{|c|c|c|c|c|c|c|c|c|}
\hline$N$ & $\begin{array}{c}v \\
\left(10^{6} \mathrm{~m} / \mathrm{s}\right)\end{array}$ & $\begin{array}{c}n \\
\left(10^{-3} \mathrm{e}^{-} / \text {unit cell }\right)\end{array}$ & \multicolumn{5}{|c|}{$(\mathrm{eV})$} & $\gamma_{1}$ \\
\hline \multicolumn{9}{|c|}{ ARPES [43] } \\
\hline 1 & 1.10 & 6.0 & -0.44 & -0.44 & & & & \\
\hline 2 & 1.05 & 8.1 & -0.30 & -0.35 & -0.24 & & & 0.48 \\
\hline 3 & 1.06 & 8.0 & -0.21 & -0.34 & -0.16 & -0.14 & & 0.44 \\
\hline 4 & 1.06 & 7.7 & -0.15 & -0.37 & -0.10 & -0.06 & -0.05 & 0.44 \\
\hline \multicolumn{9}{|c|}{ STS [67] } \\
\hline 2 & 1.06 & & -0.30 & -0.36 & -0.24 & & & 0.46 \\
\hline 3 & 1.06 & & -0.22 & -0.34 & -0.16 & -0.14 & & 0.44 \\
\hline 4 & 1.05 & & -0.11 & -0.28 & -0.10 & -0.03 & -0.01 & 0.44 \\
\hline
\end{tabular}

of massive Dirac Fermions leads, for example, to an unconventional integer quantum Hall effect [21]. In the vicinity of the $K$-point of the hexagonal Brillouin zone the band structure of bilayer graphene contains (see figs. 4 and 5) four parabolic bands [68]. Provided that there is no asymmetry between the two layers, two bands are degenerate at the $K$-point. The degeneracy is lifted when the symmetry between the two graphene layers is broken [68]. This can be tested experimentally by ARPES as was shown by Ohta et al. [36].

In FLG on SiC substrates (see section 4.1) the bottom graphene layers are charged due to electrons from the substrate with the carrier concentration decreasing from layer to layer. In the case of bilayer graphene, the two layers carry different amounts of charge and thus feel different on-site Coulomb potentials $E_{i}$ as sketched at the bottom of fig. 5. As a consequence a band gap opens in the electronic structure of bilayer graphene which can be observed by ARPES (see fig. 5). Adsorbed potassium atoms donate electrons into the top layer. An increasing $\mathrm{K}$ coverage eventually balances the charge in the bottom layer which results in a closing of the gap. Finally, an overweight of charge in the top layer reopens the gap. That this effect is at all visible is due to the fact that the screening length is short (see above). A similar effect was reported for graphite where a surface gap is opened upon alkali metal adsorption [71].

These results demonstrate that the electronic structure of bilayer graphene can be manipulated in a controlled manner by varying the potential across the two layers. This could be the key for a switching functionality provided one manages to arrange the potential in such a way that the Fermi level is in the band gap. Recently the successful fabrication of a bilayer switch was reported by Oostinga et al. [72] using exfoliated bilayer graphene with a bottomand a top-gate.

4.3 Quasi-particle dynamics in the electronic band structure of graphene Fig. 6 shows a closeup of the $\pi$-bands near the $K$-point of monolayer graphene on

$\mathrm{SiC}(0001)$. The data in fig. 6(a) and (b) where taken by varying $k_{\|}$perpendicular to and along the $\overline{\Gamma K}$ direction, respectively. While both branches of the $\pi$-band are visible in fig. 6(a), only one is visible in fig. 6(b). This is due to interference of emission from the two sub-lattices of graphene $[40,73,74]$. The bands deviate considerably from the expected linear behavior. This was attributed to many-body interactions such as electron-phonon, electronelectron, and electron-plasmon coupling [39,40]. This is evident for undoped graphene (see fig. 4) as well as for graphene doped with potassium $[39,40]$. Such many-body interactions are of interest for understanding the apparent superconductivity in carbon nano tubes [75, 76], alkalidoped $\mathrm{C}_{60}$ crystals [77], and graphite intercalation com-

(a)

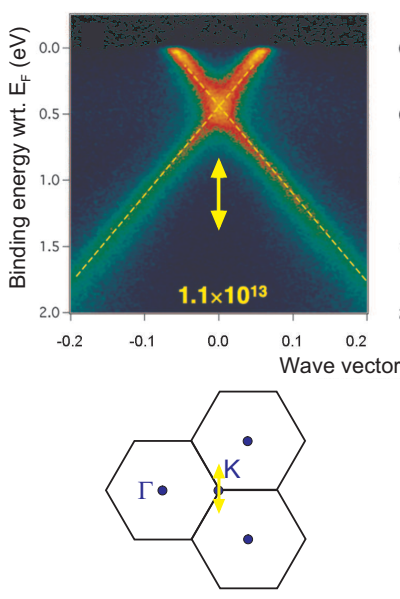

Figure 6 Experimental band structure of graphene on $\mathrm{SiC}(0001)$ near $E_{F}$ at an electron concentration of $n=1.1 \times 10^{13} \mathrm{~cm}^{-2}$ measured (a) perpendicular and (b) along $\overline{\Gamma K}$ direction $[39,40]$. Two kinks are visible. The kink at $200 \mathrm{meV}$ is due to electronphonon coupling. The second kink shifts with doping concentration and is thus caused by electronic excitations $[39,40]$. 
(a)

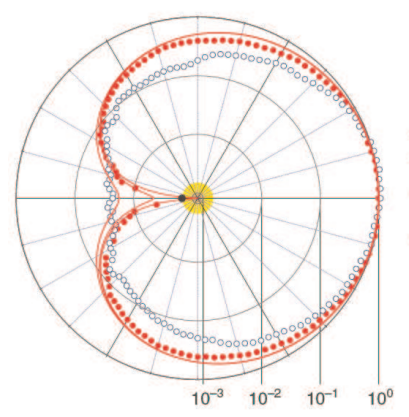

(b)

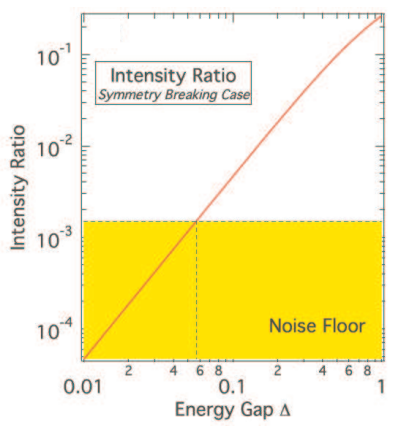

Figure 7 (a) Polar plot of the intensity at the Fermi level for monolayer (solid circles) and bilayer graphene (open circles) on $\mathrm{SiC}(0001)$. The intensity was obtained by fitting momentum distribution curves (Macs) taken along radial cuts through the $\mathrm{K}$ point of the Brillouin zone. The solid lines are theoretical intensities calculated for asymmetry parameters $\Delta=0.0,0.1,0.2 \mathrm{eV}$ using the formalism of Shirley [73]. The solid black data point represents the upper limit based on the noise floor indicated by the central yellow circle. (b) Ratio of the weakest to strongest emission intensities as a function of asymmetry parameter $\Delta$. The noise floor (yellow region) limits the asymmetry parameter $\Delta$ to be less than $55 \mathrm{meV}$. Adapted from [39]

pounds [78-80] for which graphene stands as a model system. Therefore the quasi-particle dynamics in bare and $\mathrm{K}$ doped graphene on $\mathrm{SiC}(0001)$ was analyzed in Bostwick et al. [39].

The analysis [39] showed that the kinks observed in the ARPES measurements are caused by a complicated energy dependence of the scattering rate. Three different decay channels are necessary to account for the experimentally observed spectral function: (i) electron-phonon coupling; (ii) electron-hole pair creation; (iii) electron plasmon coupling. It was necessary to include electron-plasmon coupling in order to model that region of the band structure $\left(E_{D} \leq E \leq 2 E_{D}\right)$ where electron-hole pair creation is unfavorable due to the small momentum change associated with that decay channel. Note that while the effect of electron-phonon coupling is independent of the doping, the electronic excitations show a clear dependence on the electron concentration, that is well reproduced within the model $[39,40]$. The results of that work [39] show also that the quasi-particle behavior of the charge carriers is not affected by the fact that they have to be treated as Dirac fermions in graphene.

4.4 Substrate-induced band gap in epitaxial graphene on $\mathrm{SiC}(0001)$ ? Recently, it was suggested that the deviation of the $\pi$-bands from the linear dispersion in the vicinity of the Dirac-point $E_{D}$ is not due to the above mentioned many-particle interactions but is caused instead by a breaking of the AB symmetry in epitaxial graphene on $\mathrm{SiC}(0001)$ [81-83] due to interaction with the substrate. It was proposed that this symmetry breaking causes a band gap of $0.26 \mathrm{eV}$ in graphene on $\mathrm{SiC}(0001)$ [81-83].

There are several arguments against that scenario $[40$, 84]. STS measurements on graphene do not reveal a band gap at the Dirac point $[67,85]$ and images show little indication of the proposed symmetry breaking. The strongest arguments, however, are the clear doping dependence of the observed electronic contributions to the scattering rate [39], which is supported by theoretical results [86-88], and the anisotropy of the photoelectron intensity of the $\pi$-bands in the vicinity of the K-point. The intensity variations around the $K$-point provides an excellent test for the presence of an asymmetry between A and B-sites of graphene $[40,74]$. Such an analysis was carried out in Bostwick et al. [40] and the result is shown in figure 7(a) where the experimental intensity distribution of the Fermi surface of graphene on $\mathrm{SiC}(0001)$ is compared to theoretical models including an asymmetry in the potentials on the two sublattices of $\Delta=0.0,0.1,0.2 \mathrm{eV}$ which should lead to gaps of the same size. As discussed elsewhere [39] this analysis limits the gap to a maximum value of $0.055 \mathrm{eV}$, which is more than five times smaller than the value suggested by Zhou et al. [81-83] $(0.26 \mathrm{eV})$. Therefore, the interpretation of the spectral function of graphene on $\mathrm{SiC}$ in terms of manybody effects [39] is correct.

5 Summary and outlook The present paper has given an overview over recent studies on the properties of epitaxial graphene primarily focusing on the results of photoelectron spectroscopy measurements. It is evident that this method has been able to provide detailed information about different aspects of epitaxial graphene.

Epitaxial graphene is a remarkable material with a large potential for application. In order to reach that goal, further work is required in order to complete our understanding of this material, the improvement of its production, its interplay with other materials such as metals and oxides, and its functionalization. Photoemission will continue to be a useful tool in all these areas.

Acknowledgements This work was supported by the BMBF under contract number $05 \mathrm{ES} 3 \mathrm{XBA} / 5$, by the U.S. Department of Energy, Office of Basic Sciences under Contract No.DEAC02-05CH11231, by the Max Planck Society and the European Science Foundation under the EUROCORES SONS program, by the DFG under the auspices of the DFG research unit "SiC als Halbleitermaterial: Alternative Wege in Zchtung und Dotierung", and by the Bavaria California Technology Center.

\section{References}

[1] The Lemelson-MIT Program, Edward Acheson - Carborundum, electronic source (http://web.mit.edu/invent/iow/acheson.html).

[2] S. A. Reshanov, K. Emtsev, F. Speck, K. Y. Gao, T. Seyller, G. Pensl, and L. Ley, Phys. Stat. Sol. same volume, ??? (2008).

[3] C. Berger, Z. M. Song, T. B. Li, X. B. Li, A. Y. Ogbazghi, R. Feng, Z. T. Dai, A. N. Marchenkov, E. H. Conrad, P. N. 
First, and W. A. de Heer, J. Phys. Chem. B 108, 19912 (2004).

[4] C. Berger, Z. M. Song, X. B. Li, X.S. Wu, N. Brown, C. Naud, D. Mayo, T. B. Li, J. Hass, A. N. Marchenkov, E. H. Conrad, P. N. First, and W. A. de Heer, Science 312, 1191 (2006).

[5] W. A. de Heer, C. Berger, X. Wu, P. N. First, E. H. Conrad, X. Li, T. Li, M. Sprinkle, J. Hass, M. L. Sadowski, M. Potemski, and G. Martinez, Solid State Communications 143, 92 (2007)

[6] C. Berger, Z. Song, X. Li, X. Wu, N. Brown, D. Maud, C. Naud, and W. A. de Heer, Phys. Stat. Sol. A 204, 1521 (2007).

[7] K. V. Emtsev, F. Speck, T. Seyller, J. D. Riley, and L. Ley, Phys. Rev. B 77, 155303 (2008).

[8] H. W. Kroto, J. R. Heath, S. C. Obrien, R. F. Curl, and R. E. Smalley, Nature 318, 162 (1985).

[9] S. Iijima, Nature 354, 6348 (1991).

[10] A. Airo, Fullerene (Klett, 1996).

[11] M. S. Dresselhaus, P. C. Eklund, and G. Dresselhaus (eds.), Science of Fullerenes and Carbon Nanotubes: Their Properties and Applications (Academic Press Inc., 1996).

[12] R. Saito, G. Dresselhaus, and M. S. Dresselhaus, Physical Properties of Carbon Nanotubes (Imperial College Press, 1998).

[13] S. Reich, C. Thomsen, and J. Maultzsch, Carbon Nanotubes (Wiley-VCH, 2004).

[14] P. Wallace, Phys. Rev. 71, 622 (1947).

[15] J. W. McClure, Phys. Rev. 104, 666 (1956).

[16] J. C. Slonczewski and P. R. Weiss, Phys. Rev. 109, 272 (1958).

[17] K. S. Novoselov, A. K. Geim, S. V. Morozov, D. Jiang, Y. Zhang, S. V. Dubonos, I. V. Grigorieva, and A. A. Firsov, Science 306, 666 (2004).

[18] K. S. Novoselov, D. Jiang, F. Schedin, T. J. Booth, V. V. Khotkevich, S. V. Morozov, and A. K. Geim, Proccedings of the National Academy of Science 102, 10451 (2005).

[19] K. S. Novoselov, A. K. Geim, S. V. Morozov, D. Jiang, M. I. Katsnelson, I. V. Grigorieva, S. V. Dubonos, and A. A. Firsov, Nature 438, 197 (2005).

[20] Y. Zhang, Y.W. Tan, H. L. Stormer, and P. Kim, Nature 438, 201 (2005).

[21] K. S. Novoselov, E. McCann, S. V. Morozov, V. I. Falko, M. I. Katsnelson, U. Zeitler, D. Jiang, F. Schedin, and A. Geim, Nature Physics 6, 177 (2006).

[22] K. S. Novoselov, Z. Jiang, Y. Zhang, S. V. Morozov, H. L. Stormer, U. Zeitler, J. C. Maan, G. S. Boebinger, P. Kim, and A. K. Geim, Science 315, 1379 (2007).

[23] A.C. Neto, F. Guinea, N. Peres, K. Novoselov, and A. Geim, Rev. Modern Physics (2008 (submitted)).

[24] A. K. Geim and K. Novolselov, Nature Materials 6, 183 (2007).

[25] K. Bolotin, K. Sikes, Z. Jiang, G. Fudenberg, J. Hone, P. Kim, and H. Stormer, arXiv:0802.2389v1 (2008).

[26] X. Du, I. Skachko, A. Barker, and E. Andrei, arXiv:0802.2933v1 (2008).

[27] S. V. Morozov, K. S. Novoselov, M. I. Katsnelson, F. Schedin, D. C. Elias, J. A. Jaszczak, and A. K. Geim, Phys. Rev. Lett. 100, 016602 (2008).
[28] F. Schedin, A. K. Geim, S. V. Morozov, E. W. Hill, P. Blake, M. I. Katsnelson, and K. S. Novoselov, Nat. Mater. 6 6, 652 (2007).

[29] T. Wehling, K. Novoselov, S. Morozov, E. Vdovin, M. Katsnelson, A. Geim, and A. Lichtenstein, Nano Letters 8, 173 (2008).

[30] Y. W. Son, M. Cohen, and S. Louie, Nature 444 444, 347 (2006).

[31] B. Trauzettel, D. Bulaev, D. Loss, and G. Burkard, Nature Physics 3, 192 (2007).

[32] T. Yokoyama, Phys. Rev. B 77, 073413 (2008).

[33] V. Fal'ko, Nature Physics 3, 151 (2007).

[34] T. Seyller, K. V. Emtsev, K. Gao, F. Speck, L. Ley, A. Tadich, L. Broekman, J.D. Riley, R.C.G. Leckey, O. Rader, A. Varykhalov, and A. M. Shikin, Surf. Sci. 600, 3906 (2006).

[35] T. Seyller, K. Emtsev, F. Speck, K. Y. Gao, and L. Ley, Appl. Phys. Lett. 88, 242103 (2006).

[36] T. Ohta, A. Bostwick, T. Seyller, K. Horn, and E. Rotenberg, Science 313, 951 (2006).

[37] T. Seyller, K. Emtsev, F. Speck, K. Y. Gao, and L. Ley, Materials Science Forum 556-557, 701 (2007).

[38] K. V. Emtsev, T. Seyller, F. Speck, L. Ley, P. Stojanov, J. Riley, and R. Leckey, Mater. Sci. Forum 556-557, 525 (2007).

[39] A. Bostwick, T. Ohta, T. Seyller, K. Horn, and E. Rotenberg, Nature Phys. 3(1), 36 (2007).

[40] A. Bostwick, T. Ohta, J. L. McChesney, K. V. Emtsev, T. Seyller, K. Horn, and E. Rotenberg, N. J. Phys. 9, 385 (2007).

[41] A. Bostwick, T. Ohta, J. L. McChesney, T. Seyller, K. Horn, and E. Rotenberg, The European Physical Journal - Special Topics 148, 5 (2007).

[42] A. Bostwick, T. Ohta, J.L. McChesney, T. Seyller, K. Horn, and E. Rotenberg, Solid State Comm. 143, 63 (2007).

[43] T. Ohta, A. Bostwick, J. McChesney, T. Seyller, K. Horn, and E. Rotenberg, Phys. Rev. Lett. 98, 206802 (2007).

[44] U. Starke, Atomic structure of SiC surfaces, in: Recent Major Advances in SiC, edited by W. Choyke, H. Matsunami, and G. Pensl, (Springer Scientific, 2003), p. 281.

[45] J. Bernhardt, J. Schardt, U. Starke, and K. Heinz, Appl. Phys. Lett. 74, 1084 (1999).

[46] T. Ohta, F. El Gabaly, A. Bostwick, J. McChesney, K. V. Emtsev, A. K. Schmid, T. Seyller, K. Horn, and E. Rotenberg, New J. Phys. 10, 023034 (2008).

[47] A. J. Van Bommel, J.E. Crombeen, and A. Van Tooren, Surf. Sci. 48, 463 (1975).

[48] L. I. Johansson, F. Owman, and P. Mårtensson, Phys. Rev. B 53, 13793 (1996).

[49] I. Forbeaux, J. M. Themlin, and J. M. Debever, Phys. Rev. B 58, 16396 (1998).

[50] L. I. Johansson, P. A. Glans, and N. Hellgren, Surf. Sci. 405, 288 (1998).

[51] I. Forbeaux, J. Themlin, and J. Debever, Surf. Sci. 442, 9 (1999).

[52] U. Starke, M. Franke, J. Bernhardt, J. Schardt, K. Reuter, and K. Heinz, Mater. Sci. Forum 264-268, 321 (1998).

[53] U. Starke, J. Schardt, and M. Franke, Appl. Phys. A 65, 587 (1997). 
[54] J. Hass, R. Feng, T. Li, X. Li, Z. Zong, W. A. de Heer, P. N. First, E. H. Conrad, C. A. Jeffrey, and C. Berger, App. Phys. Lett. 89, 143106 (2006).

[55] J. Hass, F. Varchon, J. E. Millan-Otoya, M. Sprinkle, W. A. de Heer, C. Berger, P. N. First, L. Magaud, and E. H. Conrad, Phys. Rev. Lett. 100, 125504 (2008).

[56] M. Naitoh, M. Kitada, S. Nishigaki, N. Toyama, and F. Shoji, Surf. Rev. Lett. 10, 473 (2003).

[57] A. Seubert, D. K. Saldin, J. Bernhardt, U. Starke, and K. Heinz, J. Phys.: Condens. Matter 12, 5527 (2000).

[58] A. Seubert, J. Bernhardt, M. Nerding, U. Starke, and K. Heinz, Surf. Sci- 454-456, 45 (2000).

[59] A. Nagashima, N. Tejima, and C. Oshima, Phys. Rev. B 50, 17487 (1994).

[60] A. M. Shikin, G. V. Prudnikova, V. K. Adamchuk, F. Moresco, and K. H. Rieder, Phys. Rev. B 62, 13202 (2000).

[61] Y.S. Dedkov, M. Fonin, U. Rüdiger, and C. Laubschat, Physical Review Letters 100, 107602 (2008).

[62] A. Varykhalov, J. Snchez-Barriga, A. Shikin, C. Biswas, E. Vescovo, and O. Rader, Electronic structure and Rashba effect in quasi-free standing graphene on the monolayer Au, unpublished, 2008.

[63] A. Mattausch and O. Pankratov, Phys. Rev. Lett. 99, 076802 (2007).

[64] A. Mattausch and O. Pankratov, Mater. Sci. Forum 556557, 693 (2007).

[65] F. Varchon, R. Feng, J. Hass, X. Li, B. N. Nguyen, C. Naud, P. Mallet, J. Y. Veuillen, C. Berger, E. Conrad, and L. Magaud, Phys. Rev. Lett 99, 126805 (2007).

[66] A. Mattausch and O. Pankratov, Phys. Stat. Sol. same volume, ??? (2008).

[67] P. Lauffer, K. Emtsev, R. Graupner, T. Seyller, L. Ley, S. Reshanov, and H. Weber, Phys. Rev. B p. in print (2008).

[68] E. McCann and V. I. Fal'ko, Phys. Rev. Lett. 96, 086805 (2006).

[69] E. McCann, Phys. Rev. B 74, 161403 (2006).

[70] F. Guinea, A. H. C. Neto, and N. M. R. Peres, Phs. Rev. B 73, 245426 (2006).

[71] M. Pivetta, F. Patthey, I. Barke, H. Hovel, B. Delley, and W. D. Schneider, Phys. Rev. B 71, 165430 (2005).

[72] J. B. Oostinga, H. B. Heersche, X. Liu, A. F. Morpurgo, and L. M. K. Vandersypen, Nat. Mater. 7, 151 (2007).

[73] E. L. Shirley, L. J. Terminello, A. Santoni, and F. J. Himpsel, Phys. Rev. B 51, 13614 (1995).

[74] M. Mucha-Kruczynski, O. Tsyplyatyev, A. Grishin, E. McCann, V.I. Fal'ko, A. Bostwick, and E. Rotenberg, arXiv:0711.1129v2 (2008).

[75] Z. K. Tang, L. Zhang, N. Wang, X. X. Zhang, G. H. Wen, G. D. Li, J.N. Wang, C. T. Chan, and P. Sheng, Science 292, 2462 (2001).

[76] M. Kociak, A. Y. Kasumov, S. Guéron, B. Reulet, I. I. Khodos, Y.B. Gorbatov, V.T. Volkov, L. Vaccarini, and H. Bouchiat, Phys. Rev. Lett. 86(11), 2416-2419 (2001).

[77] A. F. Hebard, M. J. Rosseinsky, R. C. Haddon, D. W. Murphy, S.H. Glarum, T. T.M. Palstra, A.P. Ramirez, and A. R. Kortan, Nature 350, 600 (1991).

[78] N. B. Hannay, T. H. Geballe, B. T. Matthias, K. Andres, P. Schmidt, and D. MacNair, Phys. Rev. Lett. 14(7), 225226 (1965).
[79] N. Emery, C. Herold, M. d'Astuto, V. Garcia, C. Bellin, J. F. Mareche, P. Lagrange, and G. Loupias, Physical Review Letters 95(8), 087003 (2005).

[80] T.E. Weller, M. Ellerby, S. S. Saxena, R. P. Smith, and N. T. Skipper, Nature Physics 1, 39 (2005).

[81] S. Y. Zhou, G. H. Gweon, A. V. Fedorov, P. N. First, W. A. de Heer, D. H. Lee, F. Guinea, A. H. C. Neto, and A. Lanzara, Nat. Mater. 6, 770 (2007).

[82] S. Zhou, D. Siegel, A. Fedorov, and A. Lanzara, Physica E (preprint: arXiv:0801.3862v1) p. in print (2008).

[83] S. Zhou, D. Siegel, A. Fedorov, F. E. Gabaly, A. Schmid, A. C. Neto, D. H. Lee, and A. Lanzara, Nat. Mater. 7, 259 (2008).

[84] E. Rotenberg, A. Bostwick, T. Ohta, J.L. McChesney, T. Seyller, and K. Horn, Nat. Mater. 7, 258 (2008).

[85] V. W. Brar, Y. Zhang, Y. Yayon, T. Ohta, J. L. McChesney, A. Bostwick, E. Rotenberg, K. Horn, and M. F. Crommie, Applied Physics Letters 91(12), 122102 (2007).

[86] E. H. Hwang, B. Y. K. Hu, and S. Das Sarma, Phys. Rev. B 76, 115434 (2007).

[87] E. H. Hwang and S. Das Sarma, Phys. Rev. B 77, 081412 (2008).

[88] M. Polini, R. Asgari, G. Borghi, Y. Barlas, T. PeregBarnea, and A.H. MacDonald, Phys. Rev. B 77, 081411 (2008). 